\title{
SEGREGAÇÕES GAMÉTICAS DE LOCOS ISOENZÍMICOS EM PORTA-ENXERTOS DE CITROS E SUAS CONTRIBUIÇÕES ALÉLICAS NA FORMAÇÃO DE HÍBRIDOS ${ }^{(1)}$
}

\author{
HERCULANO PENNA MEDINA FILHO ${ }^{(2,4)}$; RITA BORDIGNON ${ }^{(2)}$; \\ WALTER JOSÉ SIQUEIRA ${ }^{(3)}$
}

\begin{abstract}
RESUMO
Estudaram-se os porta-enxertos de citros, Citrus limonia (limão Cravo), C. sunki, (tangerina Sunki), C. aurantium (laranja Azeda) e Poncirus trifoliata (Trifoliata) por meio de eletroforese horizontal descontínua em gel de amido quanto aos seus genótipos e suas segregações dos locos isoenzímicos Pgi-1, Pgm-1, Got1, Got-2, Prxa-1, Aps-1 e Me-1, bem como às suas contribuições alélicas a mais de 400 híbridos entre eles. Os locos Me-1 e Aps-1, embora úteis para identificação de híbridos, são homozigotos nos genitores, portanto, inadequados às análises de segregações gaméticas nos genitores e das contribuições alélicas a seus híbridos e foram, por esta razão, desconsiderados. Em Trifoliata, o loco duplicado Got-2, anteriormente descrito com genótipo MT.SS, é na verdade SS.MT, em que S é um alelo de F em Sunki (FF.MM) e de F e M em Azeda (FM.MM), enquanto os alelos M e T da duplicação são alelos de $\mathrm{M}$ do loco homozigoto MM de Azeda e Sunki, assim determinados pelo padrão de bandas dos híbridos e pela formação dos correspondentes heterodímeros intraloco. Considerando-se 27 segregações para os cinco locos estudados verificou-se a ocorrência de distorções estatisticamente significativas em seis (22\%) delas. Assumindo-se a ausência de seleção, haja vista as condições ideais de germinação em placas de Petri, a separação dos embriões e a determinação dos genótipos já no primeiro ou segundo par de folhas, os resultados refletem, provavelmente, distorção na proporção dos alelos nos gametas dos genitores, ou transmissão diferencial dos alelos, ou ainda, distinta viabilidade de seus portadores nos zigotos híbridos. Devido à co-dominância dos alelos de isoenzimas, foi possível deduzir a contribuição relativa de cada alelo dos locos heterozigotos dos genitores a partir dos genótipos das progênies híbridas. Não se observaram distorções genotípicas oriundas do genitor Trifoliata, porém ocorreram em um loco de Azeda e de Sunki e nos três locos testados de Cravo. Tais distorções acentuadas são congruentes com uma possível origem híbrida interespecífica do limão Cravo e também ilustram a dificuldade de trabalhos de genética que envolvem quantificações de genótipos em progênies zigóticas de citros.
\end{abstract}

Palavras-chave: Citrus, genética, isoenzimas, loco duplicado, Poncirus, segregações distorcidas.

\section{ABSTRACT \\ GAMETIC SEGREGATIONS OF ISOZYME LOCI IN CITRUS ROOSTOCKS AND THEIR ALLELIC CONTRIBUTIONS IN THE FORMATION OF HYBRIDS}

The rootstock clones Rangpur lime (Citrus limonia), Sunki mandarin (C. sunki), Sour orange (C. aurantium) and Trifoliate orange (Poncirus trifoliata) were studied by discontinuous horizontal starch gel electrophoresis as to genotypes and segregations of the isozyme loci Pgi-1, Pgm-1, Got-1, Got-2, Prxa-1,

$\left.{ }^{1}\right)$ Recebido para publicação em 11 de dezembro de 2003 e aceito em 8 de julho de 2003.

$\left(^{2}\right)$ Centro de Análise e Pesquisa do Agronegócio do Café`Alcides Carvalho`, Instituto Agronômico (IAC), Caixa Postal 28, 13001-970 Campinas (SP). E-mail: medina@iac.sp.gov.br

$\left(^{3}\right)$ Centro de Pesquisa e Desenvolvimento de Recursos Genéticos Vegetais, Instituto Agronômico (IAC).

$\left({ }^{4}\right)$ Com bolsa de produtividade em pesquisa do CNPq. 
Aps-1 and Me-1 as well as their relative allelic contributions to more than 400 hybrids between them. The loci Aps-1 and Me-1, although useful for identifying hybrids are homozygous in the genitors and consequently were disregarded in the studies of segregation and allelic contribution. In Trifoliate orange, the duplicate Got-2 locus previously described as having the genotype MT.SS is actually SS.MT being S an allele of F in Sunki (FF.MM) and of F and M in Sour orange (FM.MM). M and T of the duplication are alleles of $\mathrm{M}$ of the homozygous locus of Sour orange and Sunki as determined by the banding pattern observed in the hybrids and by the formation of the correspondent intralocus heterodimers. Among 27 segregations for the five loci investigated, statistically significant distorted segregations, occurred in six $(22 \%)$ of them. Considering the absence of selection provided by ideal conditions of germination, handling of young hybrid embryos in Petri dishes and determination of genotypes at the first or second leaf pair, the results probably reflect distortion in the relative proportion of alleles in the gametes of the genitors, differential transmission of the alleles or distinct viability of their bearers in the hybrid zygotes. Due to the codominance of the alleles it was possible, from the genotypes of the hybrids, to deduce the relative contribution of each allele of the heterozygous loci of the genitors. Genotypic distortions did not come from the Trifoliate orange, occurred in one locus of Sour orange and Sunki mandarin and was traced back to Rangpur lime in the three tested loci. Such marked distortions are congruent with a possible interspecific hybrid origin of Rangpur lime and also illustrate the difficulty of genetic studies in citrus involving genotype proportions in zygotic progenies.

Key words: Citrus, distorted segregations, duplicated locus, genetics, Poncirus.

\section{INTRODUÇÃO}

Com o objetivo de desenvolver novos portaenxertos, com base em clones que se mostraram superiores ao longo de várias décadas de experimentação nas condições edafoclimáticas da citricultura brasileira, realizaram-se cruzamentos visando associar as características favoráveis presentes no limão Cravo, na tangerina Sunki, na laranja Azeda e no Poncirus trifoliata (BORDIGNON et al., 1990; BORDIGNON, 1995) . Em tais porta-enxertos é freqüente a ocorrência de sementes poliembriônicas contendo embriões apomíticos que se desenvolvem a partir do nucelo (Medina Filho et al., 1993), gerando os clones nucelares, que são pré-requisitos para a formação de mudas uniformes.

Para fins de melhoramento, a ocorrência desses embriões adventícios releva a necessidade, após realizar polinizações controladas, de se identificar os híbridos, separando-os dos clones maternos nucelares. Esse processo pode ser realizado, em alguns casos, por marcadores morfológicos (Toxopeus, 1962; BALlvé et al., 1997), mas, na maioria das situações são utilizados marcadores isoenzímicos (BALLVÉ et al.,1991). Dessa forma, o processo de identificação de híbridos precede ao estabelecimento dos campos de seleção, pois pode ser realizado com tecidos do primeiro par de folhas de uma plântula, diminuindo o custo do programa de seleção que, a partir daí, se desenvolve por vários anos.

Plantas resultantes dos cruzamentos entre porta-enxertos elite foram submetidas à eletroforese de isoenzimas e avaliadas para vários locos. Por serem marcadores co-dominantes, foi possível realizar para os quatro porta-enxertos genitores uma análise de segregação de cada um dos locos heterozigóticos, determinando-se a contribuição relativa de cada alelo na formação dos híbridos.

\section{MATERIAL E MÉTODOS}

Realizaram-se hibridações envolvendo Poncirus trifoliata, Citrus sunki, Citrus aurantium e Citrus limonia, no Centro Avançado de Pesquisa Tecnológica do Agronegócio de Citros "Sylvio Moreira", em Cordeirópolis, (SP), enquanto os procedimentos laboratoriais (eletroforeses) foram efetuados no Centro de Pesquisa e Desenvolvimento de Recursos Genéticos Vegetais, do Instituto Agronômico, em Campinas. Procedimentos de polinizações controladas, manipulação de sementes poliembriônicas, individualização de embriões e condução de plântulas são descritos em detalhes em BORDIGNON et al. (1990), MedinA-FilHo et al. (1992; 1993) e BordignON (1995).

Determinaram-se os genótipos dos locos isoenzímicos após eletroforese horizontal descontínua em gel de amido (TANKSLEY, 1979), com revelação para os sistemas PGI (Fosfoglucoisomerase), PGM (Fosfoglucomutase), GOT (Glutamato oxalaceto transaminase), PRX (Peroxidase), ME (Enzima málica) e APS (Fosfatase ácida), conforme especificações e métodos descritos em BALlvé et al. (1991, 1995). A identificação e as abreviaturas dos clones estudados encontram-se no quadro 1.

Os valores de Qui-quadrado $\left(\chi^{2}\right)$ e suas probabilidades para as segregações genotípicas 
dos híbridos resultantes e gaméticas (alelos dos locos nos genitores) foram obtidos com a ajuda do software Minitab versão 13. Para as segregações 1:1, com apenas 1 grau de liberdade, aplicou-se a correção de Yates para continuidade (SNEDECOR e COCHRAN, 1967; Little e Hills, 1978). Para $\chi^{2}$ totais utilizaram-se $\chi^{2}$ não-corrigidos, dos quais, subtraindo-se os combinados, obtiveram-se os valores dos $\chi^{2}$ para heterogeneidade.

\section{RESULTADOS E DISCUSSÃO}

O quadro 1 apresenta os genótipos dos locos isoenzímicos Pgi-1, Pgm-1, Got-1, Got-2, Prxa-1, Me-1 e Aps-1 dos genitores e dos híbridos. Os genótipos dos genitores estão de acordo com BALLVÉ et al (1991) e o dos híbridos resultantes foram deduzidos de acordo com as segregações genéticas. Exceção é feita, entretanto, em relação ao loco duplicado Got-2 que, em Trifoliata, segundo BALLVÉ et al (1991), seria MT.SS.

Os genótipos observados nos híbridos Trifoliata x Azeda se mostraram, contudo, incompatíveis com esse genótipo MT.SS do Trifoliata, pois se observou a ocorrência somente dos genótipos FS e MS em um loco e MT e MM em outro, assim determinados pela formação dos correspondentes heterodímeros intraloco. Se o Trifoliata fosse MT.SS, seria esperado obter, desse cruzamento com Azeda (FM.MM), híbridos com quatro genótipos alternativos MT, FT, MM e FM para um loco e, para o outro, apenas o genótipo MS, o que não se observou. Portanto, S de Trifoliata é um alelo do loco cujo genótipo é FM em Azeda e o loco cujo genótipo é MT corresponde ao loco MM de Azeda. Da mesma forma, observaram-se também, genótipos não esperados nos híbridos Trifoliata x Sunki (FS.MT e FS.MM respectivamente), explicados apenas caso Trifoliata fosse SS.MT. Conclui-se que é esse o genótipo do Trifoliata Davis A, para o loco Got-2 e assim referido no quadro 1 e nas análises subseqüentes.

Permanece a interessante questão sob o ponto de vista genético e evolutivo, previamente levantada por BALlVé et al (1991), se os dois locos de Got-2 estão ligados ou se são independentes. Os cruzamentos aqui analisados não são discriminantes para se testar essas hipóteses, o que não impede, entretanto, a inequívoca identificação dos híbridos e as análises genéticas subseqüentes deste trabalho.

Com referência aos locos Me-1 e Aps-1, observou-se (Quadro 1) que, apesar de apropriados para identificar híbridos de Trifoliata, são, entretanto, inadequados ao estudo de segregações gaméticas e contribuições alélicas, por serem homozigotos em todos os genitores e, conseqüentemente, não foram considerados nas discussões que se seguem.

No quadro 2 são indicados, para cada loco, o número de híbridos de cada genótipo, os valores de Qui-quadrado $\left(\chi^{2}\right)$ para as proporções de 1:1 e 1:1:1:1 e as respectivas probabilidades. Observa-se, entre as 27 segregações estudadas, para os cinco locos isoenzímicos, a ocorrência de seis segregações com distorção $(p<0,05)$. Cabe ressaltar que não se fez nenhuma seleção dos híbridos para esse estudo.

Esses híbridos, portanto, correspondem efetivamente a todos os embriões híbridos produzidos. Após a germinação de sementes em placas de Petri, todos os embriões foram transplantados para bandejas e mantidos em casa de vegetação até o estádio do primeiro ou segundo par de folhas quando foram, então, avaliados por eletroforese de isoenzimas.

Por essa razão, as distorções nas proporções dos genótipos dos híbridos refletem distorções na proporção desses alelos, nos gametas dos genitores ou sua transmissão diferencial, ou ainda, viabilidade distinta dos seus portadores na formação ou no desenvolvimento dos zigotos híbridos.

A identificação dos genótipos dos locos isoenzímicos é inequívoca, devido às bandas apresentarem migrações diferentes nos géis e os alelos serem co-dominantes. Por esse motivo, foi possível deduzir a origem de cada alelo dos híbridos como oriundo do genitor masculino ou do feminino (Quadro 2).

Como exemplo, para o loco Pgi-1, do cruzamento Trifoliata x Azeda resultaram 30 híbridos de genótipo FS, onde o alelo $S$ foi certamente transmitido pelo genitor masculino Azeda (e não pelo feminino Trifoliata) porque o alelo F só pode ter sido oriundo do Trifoliata. De maneira semelhante, nesse mesmo cruzamento, o alelo $S$ dos nove híbridos WS foi proveniente do Trifoliata (e não de Azeda) porque o alelo W só pode ter sido originado do genitor Azeda.

Dessa forma, estudaram-se, em cada genitor, as segregações dos alelos de cada loco heterozigoto, deduzidos pelos genótipos de seus diversos híbridos. A seguir, somaram-se todos os indivíduos portadores do mesmo alelo e compararam-se com o número de portadores do alelo alternativo para o loco em questão.

Esses resultados são mostrados no quadro 3, em que são agrupadas essas comparações com os respectivos $\chi^{2}$ corrigidos (segregação de $1: 1 ; 2$ classes, 1 grau de liberdade). Nesse quadro por exemplo, as segregações de F e S de Pgi-1 do genitor Cravo, agrupadas pelos seus híbridos com Sunki e Azeda, resultaram em $228 \mathrm{~F}$ e $71 \mathrm{~S}$, valores que mostram distorção altamente significativa. 
Quadro 1. Espécies e clones de porta-enxertos genitores, seus híbridos, identificação no Banco Ativo de Germoplasma (BAG) do CAPTA-Citros, abreviaturas usadas e respectivos genótipos dos locos isoenzímicos estudados

\begin{tabular}{|c|c|c|c|c|c|c|c|c|c|}
\hline Germoplasma & Identificação no BAG & Abreviatura & $P g i-1$ & $P g m-1$ & Got-1 & Got-2 & Prxa-1 & $M e-1$ & Aps-1 \\
\hline \multicolumn{10}{|l|}{ Espécies e Clones } \\
\hline Citrus limonia & n. ${ }^{\circ} 863$ Quadra 30 & Cravo & & & & & & & \\
\hline Limão Cravo Limeira & Banco de Matrizes & $\mathrm{C}$ & FS & $\mathrm{FF}$ & FS & FS.MM & $\mathrm{MM}$ & II & $\mathrm{CC}$ \\
\hline $\begin{array}{l}\text { Citrus sunki } \\
\text { Tangerina Sunki }\end{array}$ & $\begin{array}{c}\text { n. }{ }^{\circ} 200 \text { Coleção clones velhos } \\
\text { Banco de Matrizes }\end{array}$ & $\begin{array}{l}\text { Sunki } \\
\text { S }\end{array}$ & $\mathrm{FF}$ & $\mathrm{FF}$ & SS & FF.MM & FM & II & $\mathrm{CC}$ \\
\hline $\begin{array}{l}\text { Citrus aurantium } \\
\text { Laranja Azeda São Paulo }\end{array}$ & $\begin{array}{c}\text { n. }{ }^{\circ} 244 \text { Coleção clones velhos } \\
\text { n. }{ }^{\circ} 285 \text { Quadra } 16\end{array}$ & $\begin{array}{c}\text { Azeda } \\
\text { A }\end{array}$ & WS & FS & SS & FM.MM & FS & II & $\mathrm{CC}$ \\
\hline $\begin{array}{l}\text { Poncirus trifoliata } \\
\text { Trifoliata Davis A }\end{array}$ & n. ${ }^{\circ} 848$ Quadra 30 & $\begin{array}{c}\text { Trifoliata } \\
\text { T }\end{array}$ & FS & PM & PM & SS.MT & $\mathrm{FF}$ & $\mathrm{RR}$ & Nulo \\
\hline
\end{tabular}

\section{Híbridos}

\begin{tabular}{|c|c|c|c|c|c|c|c|c|}
\hline \multirow{4}{*}{ Trifoliata x Azeda } & \multirow{4}{*}{$\mathrm{T} \times \mathrm{A}$} & FW & $\mathrm{FP}$ & \multicolumn{3}{|c|}{ FS.MM } & \multirow{4}{*}{ RI } & \multirow{4}{*}{$\mathrm{Cn}$} \\
\hline & & FS & PS & PS & FS.MT & FF & & \\
\hline & & WF & FM & MS & MS.MM & FS & & \\
\hline & & MS & MS & & MS.MT & & & \\
\hline \multirow{2}{*}{ Trifoliata $x$ Sunki } & \multirow{2}{*}{$\mathrm{T} \times \mathrm{S}$} & FS & $\mathrm{FP}$ & PS & FS.MT & FF & \multirow{2}{*}{ RI } & \multirow{2}{*}{$\mathrm{Cn}$} \\
\hline & & FF & MF & MS & FS.MM & FM & & \\
\hline \multirow{2}{*}{ Cravo x Sunki e recíprocos } & \multirow{2}{*}{$\mathrm{C} \times \mathrm{S}, \mathrm{S} \times \mathrm{C}$} & FF & \multirow{2}{*}{$\mathrm{FF}$} & FS & FF.MM & FM & \multirow{2}{*}{ II } & \multirow{2}{*}{$\mathrm{CC}$} \\
\hline & & FS & & SS & FS.MM & $\mathrm{MM}$ & & \\
\hline \multirow{4}{*}{ Azeda $x$ Cravo e recíprocos } & \multirow{4}{*}{$\mathrm{A} \times \mathrm{C}, \mathrm{C} \times \mathrm{A}$} & WF & & & FF.MM & & \multirow{4}{*}{ II } & \multirow{4}{*}{$\mathrm{CC}$} \\
\hline & & WS & FS & FS & FS.MM & FM & & \\
\hline & & FS & FF & SS & FM.MM & MS & & \\
\hline & & SS & & & MS.MM & & & \\
\hline \multirow{4}{*}{ Azeda x Sunki e recíprocos } & \multirow{4}{*}{$A \times S, S \times A$} & & & \multirow{4}{*}{ SS } & & FF & \multirow{4}{*}{ II } & \multirow{4}{*}{$\mathrm{CC}$} \\
\hline & & WF & FS & & FF.MM & FS & & \\
\hline & & FS & $\mathrm{FF}$ & & FM.MM & $\mathrm{FM}$ & & \\
\hline & & & & & & MS & & \\
\hline
\end{tabular}


Quadro 2. Genótipos dos locos isoenzímicos Pgi-1, Pgm-1, Got-1, Got-2 e Prxa-1 dos genitores Sunki, Cravo, Trifoliata, Azeda e de seus híbridos. As letras subscritas s, c, t, a, indicam a correspondência dos alelos nos genitores e híbridos. São indicados o número de híbridos de cada genótipo resultante, o valor do Qui-quadrado $\left(\boldsymbol{\chi}^{2}\right)$ para as proporções genotípicas 1:1 com (C) e sem (S) correção de Yates e 1:1:1:1, com as respectivas probabilidades

\begin{tabular}{|c|c|c|c|c|c|c|c|c|}
\hline \multicolumn{3}{|l|}{ Loco } & \multicolumn{4}{|c|}{ Híbridos } & \multirow[t]{2}{*}{$\chi^{2}$} & \multirow[t]{2}{*}{$\mathrm{P}(\%)$} \\
\hline \multicolumn{7}{|l|}{$P g i-1$} & & \\
\hline & & & & $\mathrm{F}_{\mathrm{s}} \mathrm{F}_{\mathrm{c}}$ & $\mathrm{F}_{\mathrm{s}} \mathrm{S}_{\mathrm{c}}$ & & & \\
\hline Sunki & $x$ & Cravo & & 172 & 34 & & $91,11(\mathrm{C})$ & 0,0 \\
\hline $\mathrm{F}_{\mathrm{s}} \mathrm{F}_{\mathrm{s}}$ & & $\mathrm{F}_{c} \mathrm{~S}_{\mathrm{c}}$ & & & & & $92,45(\mathrm{~S})$ & 0,0 \\
\hline Cravo & $x$ & Sunki & & 25 & 24 & & $0,00(\mathrm{C})$ & 100 \\
\hline \multirow[t]{2}{*}{$\underline{\mathrm{F}_{\mathrm{c}} \mathrm{S}_{\mathrm{c}}}$} & & $\mathrm{F}_{\mathrm{s}} \mathrm{F}_{\mathrm{s}}$ & & & & & $0,02(\mathrm{~S})$ & 100 \\
\hline & & & & $\mathrm{F}_{\mathrm{s}} \mathrm{F}_{\mathrm{t}}$ & $\mathrm{F}_{\mathrm{s}} \mathrm{S}_{\mathrm{t}}$ & & & \\
\hline Trifoliata & $x$ & Sunki & & 6 & 7 & & $0,00(\mathrm{C})$ & 100 \\
\hline \multirow[t]{2}{*}{$\mathrm{F}_{\mathrm{t}} \mathrm{S}_{\mathrm{t}}$} & & $\mathrm{F}_{\mathrm{S}} \mathrm{F}_{\mathrm{S}}$ & & & & & $0,07(\mathrm{~S})$ & 100 \\
\hline & & & & $\mathrm{F}_{\mathrm{s}} \mathrm{S}_{\mathrm{a}}$ & $\mathrm{W}_{\mathrm{a}} \mathrm{F}_{\mathrm{s}}$ & & & \\
\hline Sunki & $x$ & Azeda & & 50 & 43 & & $0,39(\mathrm{C})$ & 100 \\
\hline \multirow[t]{2}{*}{$\mathrm{F}_{\mathrm{s}} \mathrm{F}_{\mathrm{s}}$} & & $\mathrm{W}_{\mathrm{a}} \mathrm{S}_{\mathrm{a}}$ & & & & & $0,53(\mathrm{~S})$ & 31,9 \\
\hline & & & $\mathrm{F}_{\mathrm{c}} \mathrm{S}_{\mathrm{a}}$ & $\mathrm{W}_{\mathrm{a}} \mathrm{F}_{\mathrm{c}}$ & $\mathrm{W}_{\mathrm{a}} \mathrm{S}_{\mathrm{c}}$ & $\mathrm{S}_{\mathrm{a}} \mathrm{S}_{\mathrm{c}}$ & & \\
\hline Cravo & $x$ & Azeda & 21 & 10 & 7 & 6 & 12,91 & 0,5 \\
\hline \multirow[t]{2}{*}{$\mathrm{F}_{\mathrm{c}} \mathrm{S}_{\mathrm{c}}$} & & $\mathrm{W}_{\mathrm{a}} \mathrm{S}_{\mathrm{a}}$ & & & & & & \\
\hline & & & $\mathrm{F}_{\mathrm{t}} \mathrm{S}_{\mathrm{a}}$ & $\mathrm{W}_{\mathrm{a}} \mathrm{F}_{\mathrm{t}}$ & $\mathrm{W}_{\mathrm{a}} \mathrm{S}_{\mathrm{t}}$ & $\mathrm{S}_{\mathrm{a}} \mathrm{S}_{\mathrm{t}}$ & & \\
\hline Trifoliata & $x$ & Azeda & 30 & 5 & 9 & 12 & 26,14 & 0,0 \\
\hline $\mathrm{F}_{\mathrm{t}} \mathrm{S}_{\mathrm{t}}$ & & $\mathrm{W}_{\mathrm{a}} \mathrm{S}_{\mathrm{a}}$ & & & & & & \\
\hline \multicolumn{9}{|l|}{ Pgm-1 } \\
\hline & & & & $\mathrm{F}_{\mathrm{c}} \mathrm{F}_{\mathrm{a}}$ & $\mathrm{F}_{\mathrm{c}} \mathrm{S}_{\mathrm{a}}$ & & $3,20(\mathrm{C})$ & 7,9 \\
\hline \multirow{3}{*}{$\begin{array}{l}\text { Cravo } \\
\mathrm{F}_{\mathrm{c}} \mathrm{F}_{\mathrm{c}} \\
\end{array}$} & $x$ & Azeda & & 29 & 16 & & $3,76(S)$ & 4,3 \\
\hline & & $\mathrm{F}_{\mathrm{a}} \mathrm{S}_{\mathrm{a}}$ & & & & & & \\
\hline & & & & $\mathrm{F}_{\mathrm{s}} \mathrm{F}_{\mathrm{a}}$ & $\mathrm{F}_{\mathrm{s}} \mathrm{S}_{\mathrm{a}}$ & & & \\
\hline \multirow{3}{*}{$\begin{array}{l}\text { Sunki } \\
\mathrm{F}_{\mathrm{S}} \mathrm{F}_{\mathrm{s}}\end{array}$} & $x$ & Azeda & & 32 & 28 & & $0,15(\mathrm{C})$ & 100 \\
\hline & & $\mathrm{F}_{\mathrm{a}} \mathrm{S}_{\mathrm{a}}$ & & & & & $0,27(S)$ & 100 \\
\hline & & & & $\mathrm{F}_{\mathrm{s}} \mathrm{P}_{\mathrm{t}}$ & $\mathrm{F}_{\mathrm{s}} \mathrm{M}_{\mathrm{t}}$ & & & \\
\hline \multirow{3}{*}{$\begin{array}{l}\text { Trifoliata } \\
\mathrm{P}_{\mathrm{t}} \mathrm{M}_{\mathrm{t}}\end{array}$} & $x$ & Sunki & & 9 & 4 & & $1,23(\mathrm{C})$ & 31,9 \\
\hline & & $\mathrm{F}_{\mathrm{S}} \mathrm{F}_{\mathrm{s}}$ & & & & & $1,92(\mathrm{~S})$ & 15,3 \\
\hline & & & $\mathrm{F}_{\mathrm{a}} \mathrm{P}_{\mathrm{t}}$ & $\mathrm{F}_{\mathrm{a}} \mathrm{M}_{\mathrm{t}}$ & $\mathrm{M}_{\mathrm{t}} \mathrm{S}_{\mathrm{a}}$ & $\mathrm{P}_{\mathrm{t}} \mathrm{S}_{\mathrm{a}}$ & & \\
\hline \multirow{2}{*}{\multicolumn{2}{|c|}{$\begin{array}{l}\text { Trifoliata } \mathrm{x} \\
\mathrm{P}_{\mathrm{t}} \mathrm{M}_{\mathrm{t}}\end{array}$}} & Azeda & 19 & 14 & 13 & 10 & 3,0 & 39,3 \\
\hline & & $\mathrm{F}_{\mathrm{a}} \mathrm{S}_{\mathrm{a}}$ & & & & & & \\
\hline
\end{tabular}

\section{Got-1}

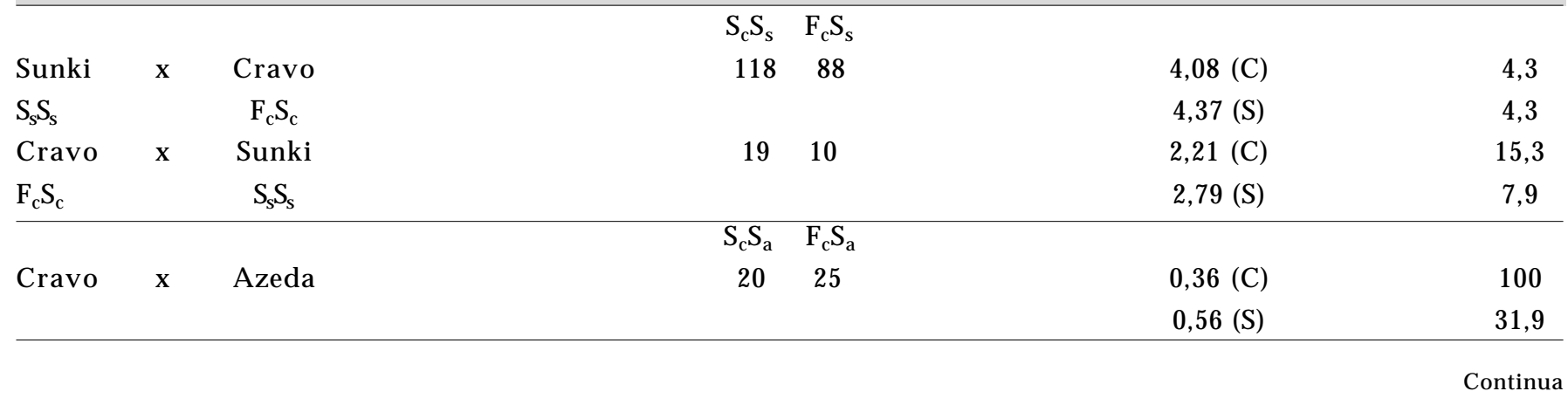


Quadro 2. Conclusão

\begin{tabular}{|c|c|c|c|c|c|}
\hline \multicolumn{2}{|l|}{ Loco } & \multicolumn{2}{|c|}{ Híbridos } & \multirow[t]{2}{*}{$\chi^{2}$} & \multirow[t]{2}{*}{$\mathrm{P}(\%)$} \\
\hline & & $\mathrm{P}_{\mathrm{t}} \mathrm{S}_{\mathrm{s}}$ & $\mathrm{M}_{\mathrm{t}} \mathrm{S}_{\mathrm{s}}$ & & \\
\hline Trifoliata $\mathrm{x}$ & Sunki & 5 & 9 & $0,64(\mathrm{C})$ & 31,9 \\
\hline $\mathrm{P}_{\mathrm{t}} \mathrm{M}_{\mathrm{t}}$ & $\mathrm{S}_{\mathrm{S}} \mathrm{S}_{\mathrm{S}}$ & & & $1,14(\mathrm{~S})$ & 31,9 \\
\hline & & $\mathrm{P}_{\mathrm{t}} \mathrm{S}_{\mathrm{a}}$ & $\mathrm{M}_{\mathrm{t}} \mathrm{S}_{\mathrm{a}}$ & & \\
\hline Trifoliata $x$ & Azeda & 13 & 10 & $0,17(\mathrm{C})$ & 100 \\
\hline$P_{t} M_{t}$ & $\mathrm{~S}_{\mathrm{a}} \mathrm{S}_{\mathrm{a}}$ & & & $0,39(\mathrm{~S})$ & 100 \\
\hline
\end{tabular}

\section{Got-2}

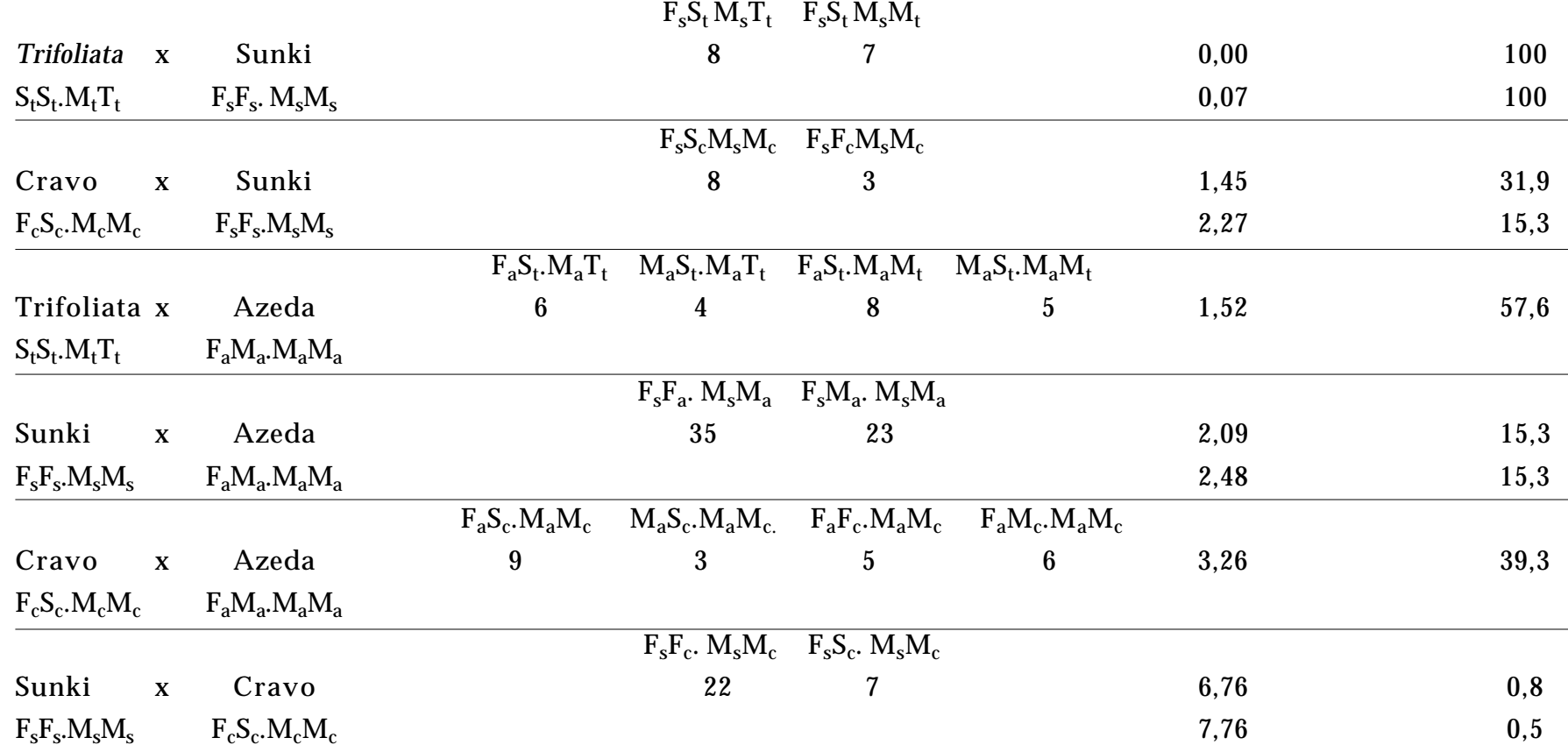

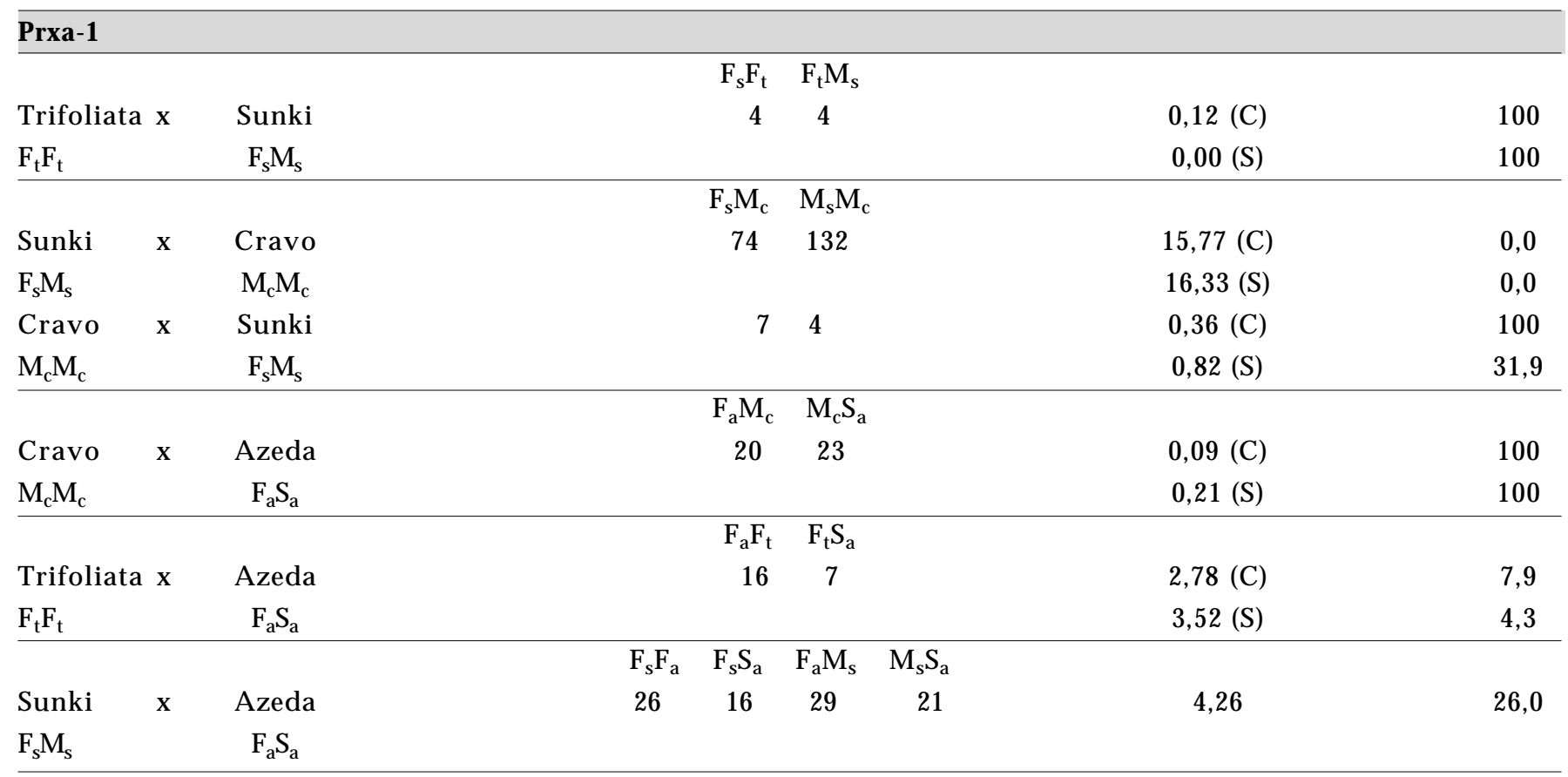


Quadro 3. Segregações gaméticas em locos isoenzímicos heterozigotos nos porta-enxertos genitores Cravo, Azeda, Sunki e Trifoliata. As letras subscritas s, c, t, a, indicam a correspondência dos alelos do genitor em seus híbridos. É indicado o número de híbridos resultantes agrupados de acordo com as contribuições alélicas dos genitores. Valores de Qui-quadrado $\left(\boldsymbol{\chi}^{2}\right)$ com (C) e sem (S) a correção de Yates e respectivas probabilidades

\begin{tabular}{|c|c|c|c|c|c|}
\hline Genitor & Loco & \multicolumn{2}{|c|}{ Híbridos } & $\chi^{2}$ & $\mathrm{P}(\%)$ \\
\hline \multicolumn{6}{|l|}{ CRAVO } \\
\hline $\begin{array}{cc}\text { Sunki } & \text { Cravo } \\
\text { FF } & \mathrm{F}_{\mathrm{c}} \mathrm{S}_{\mathrm{c}}\end{array}$ & $\operatorname{Pg} i-1$ & $\begin{array}{l}\mathrm{FF}_{\mathrm{c}} \\
172\end{array}$ & $\begin{array}{l}\mathrm{FS}_{\mathrm{c}} \\
34\end{array}$ & $\begin{array}{l}91,11(\mathrm{C}) \\
92,45(\mathrm{~S})\end{array}$ & $\begin{array}{l}0,0 \\
0,0\end{array}$ \\
\hline $\begin{array}{rrc}\text { Cravo } & \text { Sunki } \\
\mathrm{F}_{\mathrm{c}} \mathrm{S}_{\mathrm{c}} & \mathrm{FF}\end{array}$ & Pgi-1 & $\begin{array}{l}\mathrm{FF}_{\mathrm{c}} \\
25\end{array}$ & $\begin{array}{l}\mathrm{FS}_{\mathrm{c}} \\
24\end{array}$ & $\begin{array}{l}0,00(\mathrm{C}) \\
0,02(\mathrm{~S})\end{array}$ & $\begin{array}{l}100 \\
100\end{array}$ \\
\hline 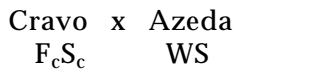 & $\operatorname{Pg} i-1$ & $\begin{array}{c}\mathrm{F}_{\mathrm{c}} \mathrm{S}+\mathrm{WF}_{\mathrm{c}} \\
31\end{array}$ & $\begin{array}{c}\mathrm{WS}_{\mathrm{c}}+\mathrm{S}_{\mathrm{c}} \mathrm{S} \\
13\end{array}$ & $\begin{array}{l}6,57(\mathrm{C}) \\
7,36(\mathrm{~S})\end{array}$ & $\begin{array}{l}0,8 \\
0,8\end{array}$ \\
\hline Total & Pgi-1 & $228\left(\mathrm{~F}_{\mathrm{c}}\right)$ & $71\left(\mathrm{~S}_{\mathrm{c}}\right)$ & $\begin{array}{l}81,39(\mathrm{C}) \\
82,44(\mathrm{~S})\end{array}$ & $\begin{array}{l}0,0 \\
0,0\end{array}$ \\
\hline 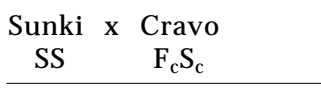 & Got-1 & $\begin{array}{l}\mathrm{S}_{\mathrm{c}} \mathrm{S} \\
118\end{array}$ & $\begin{array}{l}\mathrm{F}_{\mathrm{c}} \mathrm{S} \\
88 \\
\end{array}$ & $\begin{array}{l}4,08(\mathrm{C}) \\
4,37(\mathrm{~S}) \\
\end{array}$ & $\begin{array}{l}4,3 \\
4,3 \\
\end{array}$ \\
\hline 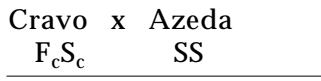 & Got-1 & $\begin{array}{l}\mathrm{S}_{\mathrm{c}} \mathrm{S} \\
20\end{array}$ & $\begin{array}{l}\mathrm{F}_{\mathrm{c}} \mathrm{S} \\
25\end{array}$ & $\begin{array}{l}0,36(\mathrm{C}) \\
0,56(\mathrm{~S})\end{array}$ & $\begin{array}{l}100 \\
31,9 \\
\end{array}$ \\
\hline $\begin{array}{rcc}\text { Cravo } & \text { Sunki } \\
\mathrm{F}_{c} \mathrm{~S}_{\mathrm{c}} & \text { SS } \\
\end{array}$ & Got-1 & $\begin{array}{l}\mathrm{S}_{\mathrm{c}} \mathrm{S} \\
19\end{array}$ & $\begin{array}{l}\mathrm{F}_{\mathrm{c}} \mathrm{S} \\
10\end{array}$ & $\begin{array}{l}2,21(\mathrm{C}) \\
2,79(\mathrm{~S})\end{array}$ & $\begin{array}{c}15,3 \\
7,9 \\
\end{array}$ \\
\hline Total & Got-1 & $157\left(\mathrm{~S}_{\mathrm{c}}\right)$ & $123\left(\mathrm{~F}_{\mathrm{c}}\right)$ & $\begin{array}{l}3,89(\mathrm{C}) \\
4,13(\mathrm{~S})\end{array}$ & $\begin{array}{l}4,3 \\
4,3\end{array}$ \\
\hline $\begin{array}{lc}\text { Cravo } & \text { Sunki } \\
\mathrm{F}_{c} \mathrm{~S}_{\mathrm{c}} \cdot \mathrm{MM} & \text { FF.MM } \\
\end{array}$ & Got-2 & $\begin{array}{c}\mathrm{FS}_{\mathrm{c}} \cdot \mathrm{MM} \\
8\end{array}$ & $\begin{array}{c}\mathrm{FF}_{\mathrm{c}} \cdot \mathrm{MM} \\
3\end{array}$ & $\begin{array}{l}1,45(\mathrm{C}) \\
2,27(\mathrm{~S})\end{array}$ & $\begin{array}{l}31,9 \\
15,3\end{array}$ \\
\hline $\begin{array}{ll}\text { Cravo } \quad x & \text { Azeda } \\
\mathrm{F}_{\mathrm{c}} \mathrm{S}_{\mathrm{c}} \cdot \mathrm{MM} & \text { FM.MM }\end{array}$ & Got-2 & $\begin{array}{c}\mathrm{FS}_{\mathrm{c}} \cdot \mathrm{MM}+\mathrm{MS}_{\mathrm{c}} \cdot \mathrm{MM} \\
12\end{array}$ & $\begin{array}{c}\mathrm{F}_{\mathrm{c}} \mathrm{F} \cdot \mathrm{MM}+\mathrm{F}_{\mathrm{c}} \mathrm{M} \cdot \mathrm{MM} \\
11\end{array}$ & $\begin{array}{l}0,00(\mathrm{C}) \\
0,04(\mathrm{~S})\end{array}$ & $\begin{array}{l}100 \\
100\end{array}$ \\
\hline $\begin{array}{lll}\text { Sunki } & \text { Cravo } \\
\text { FF.MM } & \mathrm{F}_{c} \mathrm{~S}_{c} \cdot \mathrm{MM}\end{array}$ & Got-2 & $\begin{array}{l}\mathrm{FS}_{\mathrm{c}} \cdot \mathrm{MM} \\
22\end{array}$ & $\begin{array}{c}\mathrm{FF}_{\mathrm{c}} \cdot \mathrm{MM} \\
7\end{array}$ & $\begin{array}{l}6,76(\mathrm{C}) \\
7,76(\mathrm{~S})\end{array}$ & $\begin{array}{l}0,8 \\
0,5\end{array}$ \\
\hline Total & Got-2 & $42\left(S_{c}\right)$ & $21\left(\mathrm{~F}_{\mathrm{c}}\right)$ & $\begin{array}{l}6,35(\mathrm{C}) \\
7,00(\mathrm{~S})\end{array}$ & $\begin{array}{l}1,4 \\
0,8\end{array}$ \\
\hline \multicolumn{6}{|l|}{ AZEDA } \\
\hline 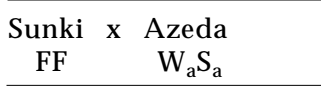 & Pgi-1 & $\begin{array}{l}\mathrm{FS}_{\mathrm{a}} \\
50\end{array}$ & $\begin{array}{c}\mathrm{W}_{\mathrm{a}} \mathrm{F} \\
43\end{array}$ & $\begin{array}{l}0,39(\mathrm{C}) \\
0,53(\mathrm{~S})\end{array}$ & $\begin{array}{l}100 \\
31,9 \\
\end{array}$ \\
\hline $\begin{array}{c}\text { Cravo } \\
\text { FS }\end{array} \quad \begin{array}{c}\text { Azeda } \\
W_{\mathrm{a}} S_{\mathrm{a}} \\
\end{array}$ & $\operatorname{Pg} i-1$ & $\begin{array}{c}\mathrm{FS}_{\mathrm{a}}+\mathrm{SS}_{\mathrm{a}} \\
27 \\
\end{array}$ & $\begin{array}{c}\mathrm{W}_{\mathrm{a}} \mathrm{F}+\mathrm{W}_{\mathrm{a}} \mathrm{S} \\
17\end{array}$ & $\begin{array}{l}1,84(\mathrm{C}) \\
2,27(\mathrm{~S})\end{array}$ & $\begin{array}{l}15,3 \\
15,3\end{array}$ \\
\hline $\begin{array}{ccc}\text { Trifoliata } & \mathrm{x} & \text { Azeda } \\
\text { FS } & & \mathrm{W}_{\mathrm{a}} \mathrm{S}_{\mathrm{a}} \\
\end{array}$ & $P g i-1$ & $\begin{array}{c}\mathrm{FS}_{\mathrm{a}}+\mathrm{SS}_{\mathrm{a}} \\
42 \\
\end{array}$ & $\begin{array}{c}\mathrm{W}_{\mathrm{a}} \mathrm{F}+\mathrm{W}_{\mathrm{a}} \mathrm{S} \\
14\end{array}$ & $\begin{array}{l}13,20(\mathrm{C}) \\
14,00(\mathrm{~S})\end{array}$ & $\begin{array}{l}0,0 \\
0,0\end{array}$ \\
\hline Total & Pgi-1 & $119\left(\mathrm{~S}_{\mathrm{a}}\right)$ & $74\left(\mathrm{~W}_{\mathrm{a}}\right)$ & $10,03(\mathrm{C})$ & 0,2 \\
\hline 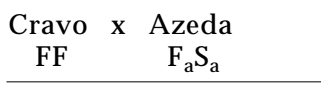 & $P g m-1$ & $\begin{array}{l}\mathrm{FF}_{\mathrm{a}} \\
29 \\
\end{array}$ & $\begin{array}{c}\mathrm{FS}_{\mathrm{a}} \\
16 \\
\end{array}$ & $\begin{array}{l}3,20(\mathrm{C}) \\
3,76(\mathrm{~S})\end{array}$ & $\begin{array}{l}7,9 \\
4,3 \\
\end{array}$ \\
\hline $\begin{array}{cc}\text { Sunki } & \text { A Azeda } \\
\text { FF } & \mathrm{F}_{\mathrm{a}} \mathrm{S}_{\mathrm{a}} \\
\end{array}$ & $P g m-1$ & $\begin{array}{l}\mathrm{FF}_{\mathrm{a}} \\
32\end{array}$ & $\begin{array}{l}\mathrm{FS}_{\mathrm{a}} \\
28\end{array}$ & $\begin{array}{l}0,15(\mathrm{C}) \\
0,27(\mathrm{~S})\end{array}$ & $\begin{array}{l}100 \\
100\end{array}$ \\
\hline $\begin{array}{ccc}\text { Trifoliata } & x & \text { Azeda } \\
\text { PM } & & F_{a} S_{a} \\
\end{array}$ & $P g m-1$ & $\begin{array}{c}\mathrm{F}_{\mathrm{a}} \mathrm{P}+\mathrm{F}_{\mathrm{a}} \mathrm{M} \\
33\end{array}$ & $\begin{array}{c}\mathrm{MS}_{\mathrm{a}}+\mathrm{PS}_{\mathrm{a}} \\
23 \\
\end{array}$ & $\begin{array}{l}1,45(\mathrm{C}) \\
1,79(\mathrm{~S}) \\
\end{array}$ & $\begin{array}{l}31,9 \\
15,2 \\
\end{array}$ \\
\hline Total & $P g m-1$ & $94\left(\mathrm{~F}_{\mathrm{a}}\right)$ & $67\left(S_{a}\right)$ & $\begin{array}{l}4,20(\mathrm{C}) \\
4,53(\mathrm{~S})\end{array}$ & $\begin{array}{l}4,3 \\
2,4 \\
\end{array}$ \\
\hline $\begin{array}{ccc}\text { Trifoliata } & x & \text { Azeda } \\
\text { SS.MT } & \mathrm{F}_{\mathrm{a}} \mathrm{M}_{\mathrm{a}} \cdot \mathrm{MM} \\
\end{array}$ & Got-2 & $\begin{array}{c}\mathrm{F}_{\mathrm{a}} \mathrm{S} \cdot \mathrm{MT}+\mathrm{F}_{\mathrm{a}} \mathrm{S} \cdot \mathrm{MM} \\
14\end{array}$ & $\begin{array}{c}\mathrm{M}_{\mathrm{a}} \mathrm{S} \cdot \mathrm{MT}+\mathrm{M}_{\mathrm{a}} \mathrm{S} \cdot \mathrm{MM} \\
9\end{array}$ & $\begin{array}{l}0,70(\mathrm{C}) \\
1,09(\mathrm{~S})\end{array}$ & $\begin{array}{l}31,9 \\
31,9 \\
\end{array}$ \\
\hline $\begin{array}{lc}\text { Sunki } & \mathrm{x} \\
\text { FF.MM } & \mathrm{F}_{\mathrm{a}} \mathrm{M}_{\mathrm{a}} \cdot \mathrm{MM}\end{array}$ & Got-2 & $\begin{array}{l}\mathrm{FF}_{\mathrm{a}} \cdot \mathrm{MM} \\
\quad 35\end{array}$ & $\begin{array}{c}\mathrm{FM}_{\mathrm{a}} \cdot \mathrm{MM} \\
23\end{array}$ & $\begin{array}{l}2,09(\mathrm{C}) \\
2,48(\mathrm{~S})\end{array}$ & $\begin{array}{l}15,3 \\
15,3\end{array}$ \\
\hline
\end{tabular}


Quadro 3. Conclusão

\begin{tabular}{|c|c|c|c|c|c|}
\hline Genitor & Loco & Híl & idos & $\chi^{2}$ & $\mathrm{P}(\%)$ \\
\hline $\begin{array}{l}\text { Cravo } x \quad \text { Azeda } \\
\text { FS.MM } \quad F_{a} M_{a} \cdot M M\end{array}$ & Got-2 & $\begin{array}{c}\mathrm{F}_{\mathrm{a}} \mathrm{S} \cdot \mathrm{MM}+\mathrm{F}_{\mathrm{a}} \mathrm{F} \cdot \mathrm{MM} \\
14\end{array}$ & $\begin{array}{c}\mathrm{M}_{\mathrm{a}} \mathrm{S} \cdot \mathrm{MM}+\mathrm{FM}_{\mathrm{a}} \cdot \mathrm{MM} \\
9\end{array}$ & $\begin{array}{l}0,70(\mathrm{C}) \\
1,09(\mathrm{~S})\end{array}$ & $\begin{array}{l}31,9 \\
31,9\end{array}$ \\
\hline Total & Got-2 & $63\left(\mathrm{~F}_{\mathrm{a}}\right)$ & $41\left(\mathrm{M}_{\mathrm{a}}\right)$ & $\begin{array}{l}4,24(\mathrm{C}) \\
4,65(\mathrm{~S})\end{array}$ & $\begin{array}{l}4,3 \\
2,4\end{array}$ \\
\hline $\begin{array}{rcc}\text { Cravo } & x & \text { Azeda } \\
\mathrm{MM} & & \mathrm{F}_{\mathrm{a}} \mathrm{S}_{\mathrm{a}} \\
\end{array}$ & Prxa-1 & $\begin{array}{c}\mathrm{F}_{\mathrm{a}} \mathrm{M} \\
20 \\
\end{array}$ & $\begin{array}{c}\mathrm{MS}_{\mathrm{a}} \\
23\end{array}$ & $\begin{array}{l}0,09(\mathrm{C}) \\
0,21(\mathrm{~S})\end{array}$ & $\begin{array}{l}100 \\
100 \\
\end{array}$ \\
\hline $\begin{array}{ccc}\text { Trifoliata } & x & \text { Azeda } \\
\text { FF } & & F_{a} S_{a}\end{array}$ & Prxa-1 & $\begin{array}{l}\mathrm{F}_{\mathrm{a}} \mathrm{F} \\
16\end{array}$ & $\begin{array}{c}\mathrm{FS}_{\mathrm{a}} \\
7\end{array}$ & $\begin{array}{l}2,78(\mathrm{C}) \\
3,52(\mathrm{~S})\end{array}$ & $\begin{array}{l}7,9 \\
4,3\end{array}$ \\
\hline 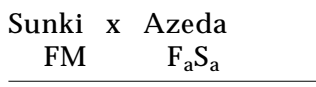 & Prxa-1 & $\begin{array}{c}\mathrm{F}_{\mathrm{a}} \mathrm{F}+\mathrm{F}_{\mathrm{a}} \mathrm{M} \\
55\end{array}$ & $\begin{array}{c}\mathrm{FS}_{\mathrm{a}}+\mathrm{MS}_{\mathrm{a}} \\
37\end{array}$ & $\begin{array}{l}3,14(\mathrm{C}) \\
3,52(\mathrm{~S})\end{array}$ & $\begin{array}{l}7,9 \\
4,3 \\
\end{array}$ \\
\hline Total & Prxa-1 & $91\left(\mathrm{~F}_{\mathrm{a}}\right)$ & $67\left(S_{a}\right)$ & $\begin{array}{l}3,35(\mathrm{C}) \\
3,65(\mathrm{~S})\end{array}$ & $\begin{array}{l}7,9 \\
4,3\end{array}$ \\
\hline SUNKI & & & & & \\
\hline $\begin{array}{cll}\begin{array}{c}\text { Trifoliata } \\
\text { FF }\end{array} & \text { Sunki } \\
\end{array}$ & Prxa-1 & $\mathrm{FF}_{\mathrm{s}}$ & $\begin{array}{c}\mathrm{FM}_{\mathrm{s}} \\
4\end{array}$ & $\begin{array}{l}0,12(\mathrm{C}) \\
0,00(\mathrm{~S})\end{array}$ & $\begin{array}{l}100 \\
100\end{array}$ \\
\hline $\begin{array}{cr}\text { Sunki } & \text { Cravo } \\
\mathrm{F}_{\mathrm{s}} \mathrm{M}_{\mathrm{s}} & \mathrm{MM} \\
\end{array}$ & Prxa-1 & $\begin{array}{c}\mathrm{F}_{\mathrm{s}} \mathrm{M} \\
74 \\
\end{array}$ & $\begin{array}{c}\mathrm{M}_{\mathrm{s}} \mathrm{M} \\
132 \\
\end{array}$ & $\begin{array}{l}15,77(C) \\
16,33(S) \\
\end{array}$ & $\begin{array}{l}0,0 \\
0,0\end{array}$ \\
\hline $\begin{array}{cr}\text { Cravo } & \text { Sunki } \\
\mathrm{MM} & \mathrm{F}_{\mathrm{s}} \mathrm{M}_{\mathrm{s}} \\
\end{array}$ & Prxa-1 & $\begin{array}{c}\mathrm{F}_{\mathrm{s}} \mathrm{M} \\
7 \\
\end{array}$ & $\begin{array}{c}\mathrm{M}_{\mathrm{s}} \mathrm{M} \\
4\end{array}$ & $\begin{array}{l}0,36(\mathrm{C}) \\
0,82(\mathrm{~S})\end{array}$ & $\begin{array}{r}100 \\
31,9\end{array}$ \\
\hline $\begin{array}{cc}\text { Sunki } & \text { Azeda } \\
\mathrm{F}_{\mathrm{s}} \mathrm{M}_{\mathrm{s}} & \text { FS } \\
\end{array}$ & Prxa-1 & $\begin{array}{c}\mathrm{F}_{\mathrm{s}} \mathrm{F}+\mathrm{F}_{\mathrm{s}} \mathrm{S} \\
42\end{array}$ & $\begin{array}{l}\mathrm{FM}_{\mathrm{s}}+\mathrm{M}_{\mathrm{s}} \mathrm{S} \\
50\end{array}$ & $\begin{array}{l}0,53(\mathrm{C}) \\
0,70(\mathrm{~S})\end{array}$ & $\begin{array}{l}31,9 \\
31,9 \\
\end{array}$ \\
\hline Total & Prxa-1 & $127\left(\mathrm{~F}_{\mathrm{s}}\right)$ & $190\left(\mathrm{M}_{\mathrm{s}}\right)$ & $\begin{array}{l}12,13(\mathrm{C}) \\
12,52(\mathrm{~S})\end{array}$ & $\begin{array}{l}0,0 \\
0,0\end{array}$ \\
\hline TRIFOLIATA & & & & & \\
\hline $\begin{array}{ccc}\text { Trifoliata } & \text { x } & \text { Sunki } \\
\mathrm{F}_{\mathrm{t}} \mathrm{S}_{\mathrm{t}} & \mathrm{FF} \\
\end{array}$ & Pgi-1 & $\begin{array}{c}\mathrm{FF}_{\mathrm{t}} \\
6\end{array}$ & $\begin{array}{c}\mathrm{FS}_{\mathrm{t}} \\
7\end{array}$ & $\begin{array}{l}0,00(\mathrm{C}) \\
0,08(\mathrm{~S})\end{array}$ & $\begin{array}{l}100 \\
100\end{array}$ \\
\hline $\begin{array}{ccc}\text { Trifoliata } & x \text { Azeda } \\
\mathrm{F}_{\mathrm{t}} \mathrm{S}_{\mathrm{t}} & \text { WS }\end{array}$ & Pgi-1 & $\begin{array}{c}\mathrm{F}_{\mathrm{t}} \mathrm{S}+\mathrm{WF}_{\mathrm{t}} \\
\quad 35\end{array}$ & $\begin{array}{c}\mathrm{WS}_{\mathrm{t}}+\mathrm{SS}_{\mathrm{t}} \\
21\end{array}$ & $\begin{array}{l}3,02(\mathrm{C}) \\
3,50(\mathrm{~S})\end{array}$ & $\begin{array}{l}7,9 \\
4,3\end{array}$ \\
\hline Total & $P g i-1$ & $41\left(\mathrm{~F}_{\mathrm{t}}\right)$ & $28\left(S_{t}\right)$ & $\begin{array}{l}2,09(\mathrm{C}) \\
2,45(\mathrm{~S})\end{array}$ & $\begin{array}{l}15,3 \\
15,3 \\
\end{array}$ \\
\hline $\begin{array}{ccc}\text { Trifoliata } & x & \text { Sunki } \\
\mathrm{P}_{\mathrm{t}} \mathrm{M}_{\mathrm{t}} & \mathrm{FF} \\
\end{array}$ & $\operatorname{Pg} m-1$ & $\begin{array}{c}\mathrm{FP}_{\mathrm{t}} \\
9\end{array}$ & $\begin{array}{c}\mathrm{FM}_{\mathrm{t}} \\
4\end{array}$ & $\begin{array}{l}1,23(\mathrm{C}) \\
1,92(\mathrm{~S})\end{array}$ & $\begin{array}{l}31,9 \\
15,3 \\
\end{array}$ \\
\hline $\begin{array}{ccc}\text { Trifoliata } & x & \text { Azeda } \\
\mathrm{P}_{\mathrm{t}} \mathrm{M}_{\mathrm{t}} & & \mathrm{FS} \\
\end{array}$ & $\operatorname{Pg} m-1$ & $\begin{array}{c}\mathrm{FP}_{\mathrm{t}}+\mathrm{P}_{\mathrm{t}} \mathrm{S} \\
29\end{array}$ & $\begin{array}{c}\mathrm{FM}_{\mathrm{t}}+\mathrm{M}_{\mathrm{t}} \mathrm{S} \\
27\end{array}$ & $\begin{array}{l}0,02(\mathrm{C}) \\
0,07(\mathrm{~S})\end{array}$ & $\begin{array}{l}100 \\
100\end{array}$ \\
\hline Total & $P g m-1$ & $38\left(\mathrm{P}_{\mathrm{t}}\right)$ & $31\left(\mathrm{M}_{\mathrm{t}}\right)$ & $\begin{array}{l}0,52(\mathrm{C}) \\
0,71(\mathrm{~S})\end{array}$ & $\begin{array}{l}31,9 \\
31,9\end{array}$ \\
\hline $\begin{array}{ccc}\text { Trifoliata } & \text { x } & \text { Sunki } \\
\mathrm{P}_{\mathrm{t}} \mathrm{M}_{\mathrm{t}} & \mathrm{SS} \\
\end{array}$ & Got-1 & $\begin{array}{c}\mathrm{P}_{\mathrm{t}} \mathrm{S} \\
5 \\
\end{array}$ & $\begin{array}{c}\mathrm{M}_{\mathrm{t}} \mathrm{S} \\
9\end{array}$ & $\begin{array}{l}0,64(\mathrm{C}) \\
1,14(\mathrm{~S}) \\
\end{array}$ & $\begin{array}{l}31,9 \\
31,9 \\
\end{array}$ \\
\hline $\begin{array}{cc}\text { Trifoliata } & \text { Azeda } \\
\mathrm{P}_{\mathrm{t}} \mathrm{M}_{\mathrm{t}} & \mathrm{SS} \\
\end{array}$ & Got-1 & $\begin{array}{l}P_{t} S \\
13\end{array}$ & $\begin{array}{c}\mathrm{M}_{\mathrm{t}} \mathrm{S} \\
10\end{array}$ & $\begin{array}{l}0,17(\mathrm{C}) \\
0,39(\mathrm{~S})\end{array}$ & $\begin{array}{l}100 \\
100\end{array}$ \\
\hline Total & Got-1 & $18\left(\mathrm{P}_{\mathrm{t}}\right)$ & $19\left(\mathrm{M}_{\mathrm{t}}\right)$ & $\begin{array}{l}0,00(\mathrm{C}) \\
0,03(\mathrm{~S})\end{array}$ & $\begin{array}{l}100 \\
100\end{array}$ \\
\hline $\begin{array}{ccc}\text { Trifoliata } & \text { Sunki } \\
\text { SS.M }_{\mathrm{t}} \mathrm{T}_{\mathrm{t}} & & \text { FF.MM } \\
\end{array}$ & Got-2 & $\begin{array}{l}\text { FS.MT }_{\mathrm{t}} \\
\quad 8\end{array}$ & $\begin{array}{l}\text { FS.MMt } \\
\quad 7\end{array}$ & $\begin{array}{l}0,00(\mathrm{C}) \\
0,07(\mathrm{~S})\end{array}$ & $\begin{array}{l}100 \\
100\end{array}$ \\
\hline $\begin{array}{cll}\text { Trifoliata } & \text { Azeda } \\
\text { SS.M }_{\mathrm{t}} \mathrm{T}_{\mathrm{t}} & \text { FM.MM } \\
\end{array}$ & Got-2 & $\begin{array}{c}\mathrm{FS}_{\mathrm{M}} \mathrm{MT}_{\mathrm{t}}+\mathrm{MS} \cdot \mathrm{MT}_{\mathrm{t}} \\
10\end{array}$ & $\begin{array}{c}\text { FS.MM } \mathrm{M}_{\mathrm{t}}+\mathrm{MS}_{13} \mathrm{MM}_{\mathrm{t}} \\
13\end{array}$ & $\begin{array}{l}0,17(\mathrm{C}) \\
0,39(\mathrm{~S})\end{array}$ & $\begin{array}{l}100 \\
100\end{array}$ \\
\hline Total & Got-2 & $18\left(\mathrm{~T}_{\mathrm{t}}\right)$ & $20\left(\mathrm{M}_{\mathrm{t}}\right)$ & $\begin{array}{l}0,03(\mathrm{C}) \\
0,10(\mathrm{~S})\end{array}$ & $\begin{array}{l}100 \\
100\end{array}$ \\
\hline
\end{tabular}


Quadro 4. Valores de qui-quadrado $\left(\boldsymbol{\chi}^{2}\right)$ total, combinado e da heterogeneidade referentes às segregações gaméticas dos alelos (entre parêntesis) dos locos isoenzímicos heterozigotos nos porta-enxertos genitores Azeda (A), Trifoliata (T), Cravo (C) e Sunki (S), deduzidas pelos genótipos de seus híbridos

\begin{tabular}{|c|c|c|c|c|c|}
\hline Genitores e híbridos & Grau de liberdade & $\chi^{2}$ & $\chi^{2}$ & $\chi^{2}$ & $\chi^{2}$ \\
\hline Azeda & & Pgi-1 (S:W) & Pgm-1 (F:S) & Got-2 (F.MM:S.MM) & Prxa-1 (F:S) \\
\hline SxA & 1 & $0,53 \mathrm{~ns}$ & $0,27 \mathrm{~ns}$ & $2,48 \mathrm{~ns}$ & $3,52 \mathrm{~ns}$ \\
\hline CxA & 1 & $2,27 \mathrm{~ns}$ & $3,76 \mathrm{~ns}$ & $1,09 \mathrm{~ns}$ & $0,21 \mathrm{~ns}$ \\
\hline TxA & 1 & 14,0 * & $1,79 \mathrm{~ns}$ & $1,09 \mathrm{~ns}$ & $3,52 \mathrm{~ns}$ \\
\hline Total & 3 & 16,80 * & $5,82 \mathrm{~ns}$ & $4,66 \mathrm{~ns}$ & $6,98 \mathrm{~ns}$ \\
\hline Combinado & 1 & 10,50 * & 4,53 * & 4,65 * & $3,65 \mathrm{~ns}$ \\
\hline Heterogeneidade & 2 & $6,3 *$ & $1,29 \mathrm{~ns}$ & $0,01 \mathrm{~ns}$ & $3,53 \mathrm{~ns}$ \\
\hline Trifoliata & & Pgi-1 (F:S) & Pgm-1 (P:M) & Got-1 (P:M) & $\begin{array}{c}\text { Got-2 } \\
\text { (SS.M:SS.T) }\end{array}$ \\
\hline $\mathrm{TxS}$ & 1 & $0,08 \mathrm{~ns}$ & $1,92 \mathrm{~ns}$ & $1,14 \mathrm{~ns}$ & $0,07 \mathrm{~ns}$ \\
\hline TxA & 1 & $3,50 \mathrm{~ns}$ & $0,07 \mathrm{~ns}$ & $0,39 \mathrm{~ns}$ & $0,29 \mathrm{~ns}$ \\
\hline Total & 2 & $3,58 \mathrm{~ns}$ & $1,99 \mathrm{~ns}$ & $1,53 \mathrm{~ns}$ & $0,36 \mathrm{~ns}$ \\
\hline Combinado & 1 & $2,45 \mathrm{~ns}$ & $0,71 \mathrm{~ns}$ & $0,03 \mathrm{~ns}$ & $0,10 \mathrm{~ns}$ \\
\hline Heterogeneidade & 1 & $1,00 \mathrm{~ns}$ & $1,13 \mathrm{~ns}$ & $1,28 \mathrm{~ns}$ & $1,50 \mathrm{~ns}$ \\
\hline Cravo & & Pgi-1 (F:S) & Got-1 (S:F) & Got-2 (S.MM:F.MM) & \\
\hline $\mathrm{SxC}$ & 1 & $92,45 *$ & $4,37 *$ & $7,76^{*}$ & \\
\hline CxS & 1 & $0,02 \mathrm{~ns}$ & $2,79 \mathrm{~ns}$ & $2,27 \mathrm{~ns}$ & \\
\hline CxA & 1 & 7,36 * & $0,56 \mathrm{~ns}$ & $0,04 \mathrm{~ns}$ & \\
\hline Total & 3 & $99,83 *$ & $7,72 \mathrm{~ns}$ & 10,07 * & \\
\hline Combinado & 1 & 82,44 * & 4,13 * & $7,00 *$ & \\
\hline Heterogeneidade & 2 & 17,39 * & $3,59 \mathrm{~ns}$ & $3,07 \mathrm{~ns}$ & \\
\hline Sunki & & $\operatorname{Prxa-1}(\mathrm{F}: \mathrm{M})$ & & & \\
\hline $\mathrm{TxS}$ & 1 & $0,00 \mathrm{~ns}$ & & & \\
\hline $\mathrm{SxC}$ & 1 & 16,33 * & & & \\
\hline CxS & 1 & $0,82 \mathrm{~ns}$ & & & \\
\hline SxA & 1 & $0,70 \mathrm{~ns}$ & & & \\
\hline Total & 4 & 17,85 * & & & \\
\hline Combinado & 1 & $12,52 *$ & & & \\
\hline Heterogeneidade & 3 & $5,33 \mathrm{~ns}$ & & & \\
\hline
\end{tabular}

${ }^{*} \mathrm{p}<0,05 ; \mathrm{ns}=$ não significativo. 
O quadro 4 mostra valores de $\chi^{2}$ não corrigidos, utilizados para o cálculo do $c^{2}$ total, do qual por subtração do $c^{2}$ combinado, obtiveram-se os valores do $c^{2}$ para heterogeneidade. Em 12 casos, 10 apresentaram valores estatisticamente não significativos, indicando a homogeneidade dos diversos grupos estudados.

Verifica-se também pelo quadro 4, que Trifoliata não apresentou distorções estatisticamente significativas, fato observado para um loco de Azeda e um de Sunki. Tais distorções ocorreram, entretanto, nos três locos testados de Cravo, o que pode ter ocorrido devido à possível estrutura genômica complexa do limão Cravo. Apesar de ser considerada uma espécie estável, Hodgson (1967) menciona que na China e no Brasil seu nome comum é limão, enquanto nos Estados Unidos e na Índia é referido como lima, porém sua denominação correta seria "tangerina ou semelhante à tangerina". Frost e Soost (1968) se referem a essa espécie como um tipo de "lima-tangerina". Segundo a classificação botânica de Swingle (SwingLe e REECE, 1967), o limão Cravo denomina-se C. reticulata var. austera, nomenclatura que se refere, evidentemente, a uma tangerina. De acordo com TANAKA (1954), a denominação válida é C. limonia, clara alusão à semelhança com os limões. A razão dessas divergências populares e entre renomadas autoridades taxonômicas pode ser devido à possível origem híbrida do limão Cravo (Bordignon, 2000).

Na maioria das espécies de Citrus existe a reprodução assexuada facultativa via semente pela embrionia nucelar, mecanismo que, certamente, favorece a manutenção de altos níveis de heterozigosidade e de estabilidade fenotípica e genotípica de indivíduos mesmo com irregularidades meióticas. Embora seja apenas uma conjectura acerca de uma das razões que levariam a distorções nas proporções dos genótipos dos híbridos investigados, é fato incontestável que tais distorções ocorreram. Esses resultados atestam a dificuldade dos estudos genéticos nos citros, principalmente quando estão envolvidas as proporções genótipicas. Esse fato vem ilustrar e corroborar considerações semelhantes a respeito da genética da tolerância à tristeza, discutidas por BoRDIGNON et al. (2004), em que as reações de tolerância e intolerância se adaptam muito bem a um modelo genético de interação epistática, porém apresentam evidentes distorções nas proporções genotípicas de alguns híbridos.

\section{CONCLUSÕES}

1. Os genótipos dos locos isoenzímicos Pgi-1, Pgm-1, Got-1, Got-2, Aps-1, Me-1 e Prxa-1 permitiram identificar cerca de 400 híbridos entre Sunki, Cravo,
Trifoliata e Azeda, analisar 27 freqüências genotípicas desses híbridos, deduzir 33 segregações gaméticas dos genitores e determinar as contribuições alélicas de cada loco heterozigoto para a formação desses híbridos.

2. O genótipo de Poncirus trifoliata Davis A para o loco duplicado Got-2 é SS.MT e não MT.SS, conforme anteriormente descrito na literatura.

3. Em 27 freqüências genotípicas estudadas nos híbridos, verificaram-se seis com distorções estatisticamente significativas. Tais distorções foram resultantes sobretudo das segregações gaméticas do limão Cravo.

4. A hipótese de que o limão Cravo (Citrus limonia Osbeck) tenha uma origem híbrida interespecífica é coerente com as segregações distorcidas observadas nessa espécie.

\section{AGRADECIMENTOS}

Os autores agradecem ao pesquisador Jorgino Pompeu Junior e Joaquim Teófilo Sobrinho, do Centro Avançado de Pesquisa Tecnológica do Agronegócios de Citros, pelas sugestões no desenvolvimento da pesquisa; a Rosa Maria L. Ballvé, pela ajuda nas análises de isoenzimas e a Silvia Luisa S. Lima, pelo auxílio na digitação.

\section{REFERÊNCIAS BIBLIOGRÁFICAS}

BALLVÉ, R.M.L.; BORDIGNON, R.; MEDINA FILHO, H.P.; SIQUEIRA, W.J.; TEÓFILO SOBRINHO, J.; POMPEU JUNIOR, J. Isoenzimas na identificação precoce de híbridos e clones nucelares no melhoramento de citros. Bragantia, Campinas, v.50, n.1, p.57-76, 1991.

BALLVÉ, R.M.L.; MEDINA FILHO, H.P.; BORDIGNON, R. Identification of reciprocal hybrids in citrus by the broadness of the leaf petiole wing. Brazilian Journal of Genetics, Ribeirão Preto, v.20, n.4, p.697-702, 1997.

BALLVÉ, R.M.L.; MEDINA FILHO, H.P.; BORDIGNON, R.; LIMA, M.M.A. Methodology for starch gel electrophoresis and protocols for isozyme of 32 plant genera. Brazilian Journal of Genetics, Ribeirão Preto, v.18, n.3, p.441-502, 1995.

BORDIGNON, R. Características agronômicas e tolerância à triste$z a$ de híbridos entre porta-enxertos elite de citros. 2000. 164f. Tese (Doutorado em Genética e Biologia Molecular). Instituto de Biologia - Universidade Estadual de Campinas. 
BORDIGNON, R. Hibridações interespecíficas, intergenéricas, intergrupais, intersubtribais, intertribais e intersubfamiliares de Citrus e gêneros relacionados. Campinas, 1995. 104f. Tese (Mestrado em Ciências Biológicas). Instituto de Biologia Universidade Estadual de Campinas.

BORDIGNON, R.; MEDINA FILHO, H.P.; BALLVÉ, R.M.L. Melhoramento genético de citros no Instituto Agronômico. Laranja, Cordeirópolis, v.11, p.167-176, 1990.

BORDIGNON, R.; MEDINA FILHO, H.P.; SIQUEIRA, W.J. Genetics of tolerance to the tristeza disease (CTV) in citrus rootstocks. Genetics and Molecular Biology, Ribeirão Preto, v.24, n.1, 2004. (no prelo)

FROST, H.B.; SOOST, R.K. Seed reproduction: development of gametes and embryos. In: REUTHER, W; BATCHELOR, L.D.; WEBBER, H.J. (Eds.). The Citrus Industry. Berkeley: University of California Press, 1968. v.2, p.290-324.

HODGSON, R.W. Horticultural varieties of citrus. In: REUTHER, W.; WEBBER, H.J.; BATCHERLOR, L.D. (Eds.) The Citrus Industry. Riverside: University of California Press, 1967. v.1, p.431-591.

LITLE, T.M.; HILLS, F.J. Agricultural experimentation: design and analysis. Santa Barbara: John Wiley, 1978. 350p.

MEDINA FILHO, H.P.; BORDIGNON, R.; BALLVÉ, R.M.L.; SIQUEIRA, W.J.; CARVALHO, M.R.T. Porta-enxertos híbridos de citros produzidos no Instituto Agronômico de Campinas. Laranja, Cordeirópolis, v.13, n.2, p.777-792, 1992.
MEDINA FILHO, H.P.; BORDIGNON, R.; BALLVÉ, R.M.L.; SIQUEIRA W.J. Genetic proof of the occurrence of mono and dizygotic hybrid twins in citrus rootstocks. Revista Brasileira de Genética, Ribeirão Preto, v.16, n.3, p.703-711, 1993.

SNEDECOR, G.W.; COCHRAN, W.G. Statistical Methods. 6.ed. Iowa: Iowa State University Press, 1967. 593p.

SWINGLE, W.T.; REECE, P.C. The botany of Citrus and its wild relatives. In: REUTHER, W.; BATCHERLOR, L.D.; WEBBER, W.J. (Ed.). The citrus industry. Riverside: University of California Press, 1967. v.1, p.190-430.

TANAKA, T. Species problem in citrus: a critical study of wild and cultivated units of citrus, based upon field studies in their native homes. Ueno, Tokyo: Japanese Society for the Promotion of Science, 1954. 152p. (Revisio aurantiacearum IX)

TANKSLEY.S.D. An efficient and economical design for starch gel electrophoresis. Report of the Tomato Cooperative, Davis, v.29, p.37-38, 1979.

TOXOPEUS, H. Notes on the genetics of a few leaf characters in the genus Citrus. Euphytica, Dordrecht, v.11, p.19-25, 1962. 